\title{
Contact problems involving a cooled punch
}

\author{
J. R. BARBER \\ University Department of Mechanical Engineering, Newcastle upon Tyne, Great Britain
}

(Received September 23, 1977)

\begin{abstract}
Certain problems in which a cooled rigid punch indents an elastic half-space have no steady state solution. A simple model is described in which it is shown that this paradox is avoided by the assumption of a thermal resistance varying inversely with contact pressure. A limiting case of this system retains linearity and introduces a state of "imperfect" contact in which contact pressure is negligible but there is significant thermal contact resistance.

This approach is generalized to permit the formulation of three dimensional contact problems and one such problem is solved for an axisymmetric geometry. Particular results are given for the identation of a half-space by a cooled rigid sphere.
\end{abstract}

\section{RÉSUMÉ}

Les problèmes de contact thermoélastiques pour un demi-espace entaillé par un poinçon rigide refroidi n'ont pas parfois de solution établie. On décrit un modèle simple demontrant qu'on évite ce paradoxe en supposant une résistance thermique varient en raison inverse du pression de contact. On retiens linearité avec un cas limitatif de ce système en introduisant une condition de contact "imparfait" ou le pression de contact est négligeable mais il y a de la résistance thermique de contact significative.

On généralise cette méthode pour permetter la formulation des problèmes de contact thermique dans trois dimensions et un problème de ce type est résolu en cas de symetrie axiale. On donne des resultats particulières pour le demi-espace entaillé par une sphère rigide refroidie.

\section{Introduction}

In thermoelastic contact problems, it is customary to assume that perfect thermal contact occurs in all regions of mechanical contact, whilst over the rest of the solid surfaces there is no heat transfer.

With this formulation, it is found that certain problems have no steady-state solution. For example, if a cooled rigid sphere is pressed into an elastic half-space, the assumption of a circular contact area leads to unacceptable tensile contact stresses near the outer radius [1]. However, the only alternative axisymmetric contact geometry is a system of concentric annuli, which is ruled out by a theorem [2] requiring that the contact area should not be multiply-connected.

In the more general case where both solids are deformable, similar problems tend 
to arise when the hotter solid has the higher thermal distortivity $(\delta)$ defined by

$$
\delta=\frac{\alpha(1+\nu)}{K},
$$

where $\alpha, K, \nu$ are respectively the coefficient of linear thermal expansion, thermal conductivity and Poisson's ratio for the material [3]. ${ }^{1}$

\section{A one-dimensional model}

The main features of the problem and the proposed solution can best be exposed by considering the simple one-dimensional system shown in Figure 1, for which $I$ am indebted to Professor Dundurs [4]. Two rigid walls, $A, B$, separated by a distance $l$ are maintained at temperatures $T_{A}, T_{B}$, respectively and a uniform elastic rod of unit cross-sectional area is built into wall $A$ as shown. The length of the rod is $\left(l-g_{0}\right)$ at the temperature $T_{0}$. Now suppose that $T_{B}$ is maintained constant at the value $T_{0}$, whilst $T_{A}$ takes various values. If the rod fails to make contact with wall $B$, the gap $g$ will be determined by

$$
g=g_{0}-\alpha \int_{0}^{l}\left(T-T_{0}\right) d x=g_{0}-\frac{\alpha Q}{\rho c},
$$

where $Q$ is the quantity of heat required to raise the temperature of the rod to this state from a datum at $T_{0}$, and $\rho, c$, are respectively the density and specific heat of the rod material.

The system will tend to a steady state in which $T=T_{A}$ throughout the rod, there being no heat loss from the end, and hence

$$
g=g_{0}-\alpha l\left(T_{A}-T_{0}\right) \text {. }
$$

The gap $g$ cannot be negative and hence we can only have continuous non-contact as long as

$\left(T_{A}-T_{0}\right) \approx g_{0} / \alpha l$.

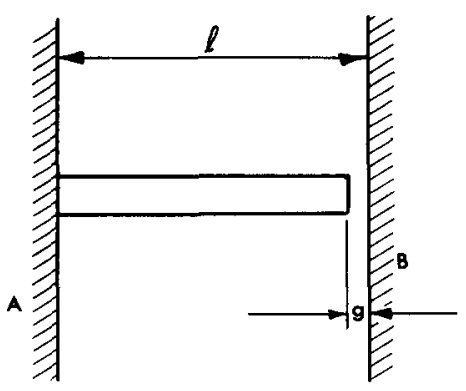

Figure 1

\footnotetext{
${ }^{1}$ If the cooler solid has the higher distortivity, there can be problems of lack of uniqueness.
} 
Now suppose the rod expands sufficiently to make contact with wall $B$. Heat will be conducted along it and in the steady-state there will be a linear variation of temperature from $T_{A}$ at one end to $T_{0}$ at the other. In this case there will be a contact pressure $p$, between the rod and wall $B$ given by

$$
p=\frac{E \alpha\left(T_{A}-T_{0}\right)}{2}-\frac{E g_{o}}{l}
$$

where $E$ is Young's modules for the material of the rod. This contact pressure cannot be negative (tensile) and hence

$$
\left(T_{A}-T_{0}\right) \geqslant 2 g_{0} / \alpha l \text {. }
$$

Considering the two inequalities $(4,6)$, we must therefore conclude that in the range

$$
g_{0} / \alpha l<\left(T_{A}-T_{0}\right)<2 g_{0} / \alpha l
$$

the system cannot exist in a steady-state either of contact or non-contact.

The absence of a uniform steady-state solution suggests that a cyclic condition will be reached in which there are alternating periods of contact and non-contact.

In such a condition, the non-contact period must follow a period of contact and the initial gap size must therefore be zero. However, during the non-contact period, the flow of heat into the rod from wall $A$ must be positive and hence, from equation (2), $d g / d t<0$-i.e. the gap can only get smaller.

It follows that contact must be re-established as soon as it is broken and it is easily shown that the contact period also can have only an infinitesimal duration. Hence, unless a physical meaning can be given to the concept of contact and non-contact alternating with infinite frequency, we must conclude that a cyclic solution is impossible.

\section{Introduction of a pressure dependent contact resistance}

A possible cause of this difficulty is the fact that the change from contact to non-contact (i.e. from zero to infinite contact resistance) is discontinuous. In a practical system, some resistance to heat flow across the contact will be experienced before the contact pressure has fallen to zero. The range of conditions over which this effect is significant may be small, but it introduces the possibility of conditions intermediate between the extremes of perfect contact and non-contact.

Suppose the system of Figure 1 is modified to include a pressure dependent contact resistance $R$.

The temperature at that end of the rod which makes contact with the wall $B$ can now differ from $T_{0}$ and will be denoted by $T_{C}$. In the steady-state, $T_{C}$ is given by

$$
\frac{K\left(T_{A}-T_{C}\right)}{l}=\frac{\left(T_{C}-T_{0}\right)}{R},
$$

where $K$ is the thermal conductivity of the rod material. 
The contact resistance, $R$, would be expected to fall as the contact pressure, $p$, rises and hence it will be provisionally ${ }^{2}$ represented in the form

$$
R=A / p
$$

where $A$ is a constant.

The contact pressure, which depends upon the mean temperature of the rod, will now be given by

$$
p=\frac{E \alpha\left(T_{A}+T_{C}-2 T_{0}\right)}{2}-E g_{0} / l
$$

(cf. equation (5) in which $T_{C}=T_{0}$ ).

Eliminating $T_{C}$ and $R$ between equations $(8,9,10)$ we obtain the relation

$$
p^{2}+\left(g_{0} / l+K A / E l-\alpha\left(T_{A}-T_{0}\right) / 2\right) E p+\left(g_{0} / l-\alpha\left(T_{A}-T_{0}\right)\right) \frac{E K A}{l}=0
$$

between $p$ and $\left(T_{A}-T_{0}\right)$, which is illustrated in Figure 2 .

Equation (11) defines a hyperbola whose asymptotes are the lines

$$
\begin{aligned}
& p=-2 A K / l, \\
& p=E \alpha\left(T_{A}-T_{0}\right) / 2-A K / l-E g_{0} / l,
\end{aligned}
$$

which are shown dotted in Figure 2. For values of $\left(T_{A}-T_{0}\right)<g_{0} / \alpha l$, both values of $p$ are negative and of no physical significance. In this range, we have continuous non-contact as indicated by the simpler model (inequality (4)). However, we now have a solution with positive contact pressure for all values of $\left(T_{A}-T_{0}\right) \geqslant g_{0} / \alpha l$,

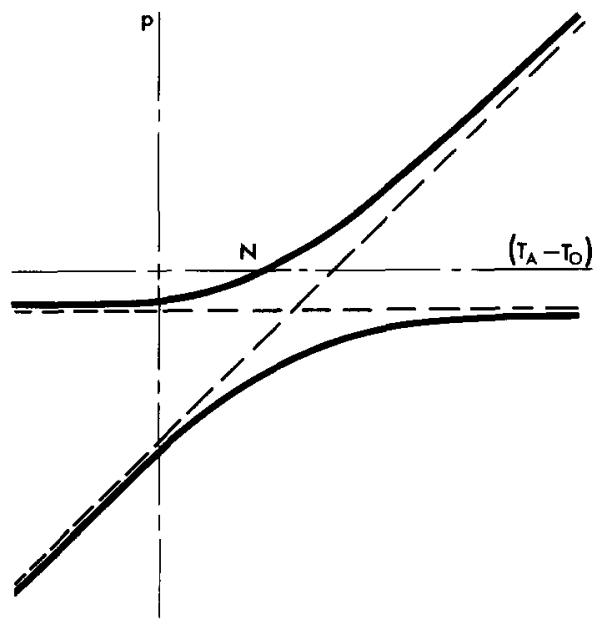

Figure 2

\footnotetext{
${ }^{2}$ In fact, the exact form of this inverse relationship proves irrelevant to the later stages of the argument.
} 
corresponding to the upper branch of the hyperbola of Figure 2 to the right of point $N$. Thus, the introduction of a pressure dependent thermal contact resistance removes the anomalies of the system of Figure 1 and permits a steady-state solution to be obtained under all applied conditions.

\section{A limiting case}

In principle, this approach could be extended directly to problems in two and three dimensions, but at the considerable cost of making the controlling integral or differential equations non-linear. However, a compromise can be achieved by examining the behaviour of the system as the constant $A$ in equation (9) becomes small.

As $A$ tends to zero, the horizontal asymptote 12 (i) in Figure 2 approaches the axis $p=0$ and the hyperbola crowds more closely into its asymptotes near their point of intersection. The point $N$ at which $p$ for the upper branch becomes positive does not move.

In the limit, the upper branch to the right of point $N$ consists of the two lines

$$
\left.\begin{array}{ll}
p=0 ; \quad 2 g_{0} / \alpha l \geqslant\left(T_{A}-T_{0}\right) \geqslant g_{0} / \alpha l, & \text { (i) } \\
p=E \alpha\left(T_{A}-T_{0}\right) / 2-E g_{0} / l ; \quad\left(T_{A}-T_{0}\right)>2 g_{0} / \alpha l . & \text { (ii) }
\end{array}\right\}
$$

The physical interpretation of this result is that, when there is a non-zero contact pressure, the thermal contact resistance is negligible and we have conditions of perfect contact (compare equations (5) and (13(ii))). However, in the range $2 \mathrm{~g}_{0} / \alpha l>$ $\left(T_{A}-T_{0}\right)>g_{0} / \alpha l$ the contact pressure is negligibly small and the contact resistance can be significant. We might describe this as a state of imperfect thermal contact. Finally, when $\left(T_{A}-T_{0}\right)<g_{0} / \alpha l$, the contact is broken and a positive gap, g, is developed as in the simple model.

It should be noted that in the state described here as imperfect contact, we know that the contact pressure is negligible (zero in the limit) and that the gap $g$ is zero, but nothing is known about the temperature of the end of the rod except that it must lie in the range $T_{A}>T_{C}>T_{0}$, since contact resistance cannot be negative. This inequality defines the conditions under which imperfect contact will occur (i.e. (13(i)) above).

\section{Thermoelastic indentation problems for the half-space}

The above approach can now be extended to problems in which a frictionless elastic half-space is indented by a perfectly conducting rigid punch. The half-space is taken to occupy the region $z>0$ and the normal displacement and traction at the surface are denoted by $u_{z}, \sigma_{z z}$ respectively, tensile tractions being considered as positive. The temperature field in the half-space is denoted by $T$. 
We now postulate that the surface of the half-space consists of regions of perfect contact, imperfect contact and non-contact, in which the boundary conditions are as follows:

(a) Perfect contact

$$
\begin{aligned}
& T=T_{0}, \\
& u_{z}=u_{0}, \\
& \sigma_{z z}<0
\end{aligned}
$$

(b) Imperfect contact

$$
\begin{aligned}
& u_{z}=u_{0} \\
& \sigma_{z z}=0 \\
& \left(T-T_{0}\right) \frac{\partial T}{\partial z}>0
\end{aligned}
$$

(c) Non-contact

$$
\begin{aligned}
& \sigma_{z z}=0, \\
& \partial T / \partial z=0, \\
& u_{z}>u_{0} ;
\end{aligned}
$$

where $u_{0}, T_{0}$ are prescribed functions describing the profile and the temperature respectively of the punch. The tangential tractions $\sigma_{x z}, \sigma_{y z}$, are taken to be zero throughout the surface. Note that those thermoelastic contact problems which can be solved with the conventional boundary conditions are included within this system as cases in which there are no regions of imperfect contact.

If the temperature field has reached a steady state, $T$ will be a harmonic function and it is possible to express the state of stress in the half-space in terms of two harmonic potential functions [5]. We note that the surface displacement $u_{z}$ and the contact stress $\sigma_{z z}$ each appear in two of the equations (14) and hence it is helpful to choose a representation in which these quantities take a particularly simple form. Such a representation can be derived from Williams' solution [5] by replacing $\phi$ by $(\psi-\chi)$ in his equations $(9-11)$, giving the solution

$$
\begin{aligned}
& u=\nabla \chi-z \nabla \partial \chi / \partial z-2(1-\nu) \nabla \psi+4(1-\nu) k \frac{\partial \psi}{\partial z}, \\
& T=\frac{2(1-\nu)}{\alpha(1+\nu)}\left\{\frac{\partial^{2} \psi}{\partial z^{2}}-\frac{\partial^{2} \chi}{\partial z^{2}}\right\}
\end{aligned}
$$

where $\boldsymbol{u}$ is the displacement vector and $\chi, \psi$ are two harmonic potential functions.

The component of stress acting on the $z$ plane is

$$
\mathbf{s}_{z} / 2 G=\boldsymbol{k} \frac{\partial^{2} \chi}{\partial z^{2}}-z \nabla \frac{\partial^{2} \chi}{\partial z^{2}},
$$

where $G$ is the modulus of rigidity. 
On the surface plane, $z=0$, this component reduces to a purely normal stress

$$
\sigma_{z z}=2 G \frac{\partial^{2} \chi}{\partial z^{2}}
$$

whilst the normal displacement at the surface is

$$
u_{z}=2(1-\nu) \frac{\partial \psi}{\partial z}
$$

from equation (15).

\section{Indentation by a cooled sphere}

To illustrate the use of these results we shall consider the case in which the half-space is indented by a cooled rigid sphere. If this problem is treated by making the conventional assumption of a dichotomy between non-contact and perfect contact, it is found that tensile contact stresses are required at the edge of the contact circle [1]. With the modified boundary conditions developed above, we should therefore expect to find an annulus of imperfect contact surrounding a central circle of perfect contact.

Denoting the inner and outer ratio of this annulus by $a, b$ respectively and using equations $(14,16,18,19)$ above, we have the following boundary conditions at the surface plane, $z=0$ :

$$
\begin{aligned}
& 0 \leqslant r \leqslant a \text { perfect contact } \\
& \partial \psi / \partial z=u / 2(1-\nu), \\
& \partial^{2} \psi / \partial z^{2}-\partial^{2} \chi / \partial z^{2}=\frac{\alpha(1+\nu) T}{2(1-\nu)}, \\
& a<r \leqslant b \text { imperfect contact } \\
& \partial \psi / \partial z=u / 2(1-\nu), \\
& \partial^{2} \chi / \partial z^{2}=0, \\
& b<r<\infty \text { non-contact } \\
& \partial^{2} \chi / \partial z^{2}=0 \\
& \partial^{3} \psi / \partial z^{3}-\partial^{3} \chi / \partial z^{3}=0
\end{aligned}
$$

where $u, T$ are prescribed functions of radius, $r$, describing the profile and the temperature respectively of the punch. 


\section{Solution}

The conditions 20 (iv, v) can be satisfied identically by representing the harmonic function $\partial^{2} \chi / \partial z^{2}$ in the form

$$
\begin{aligned}
\frac{\partial^{2} \chi}{\partial z^{2}} & =\frac{1}{2 i} \int_{0}^{a} g_{1}(t)\left\{\frac{1}{\left(r^{2}-(z+i t)^{2}\right)^{1 / 2}}-\frac{1}{\left(r^{2}+(z+i t)^{2}\right)^{1 / 2}}\right\} d t \\
& =\operatorname{Im} \int_{0}^{a} \frac{g_{1}(t) d t}{\left(r^{2}+(z-i t)^{2}\right)^{1 / 2}},
\end{aligned}
$$

where $g_{1}$, is an unknown function of $t$ to be determined from the other boundary conditions. This method of treating axisymmetric boundary value problems was developed by Green [6,7] and Collins [8].

Differentiating equation (21) with respect to $z$ we obtain

$$
\begin{aligned}
\frac{\partial^{3} \chi}{\partial z^{3}} & =\operatorname{Im} \int_{0}^{a} \frac{-(z-i t) g_{1}(t) d t}{\left(r^{2}+(z-i t)^{2}\right)^{3 / 2}} \\
& =\operatorname{Im}\left\{\frac{1}{r} \frac{d}{d r} \int_{0}^{a} \frac{(z-i t) g_{1}(t) d t}{\left(r^{2}+(z-i t)^{2}\right)^{1 / 2}}\right\} .
\end{aligned}
$$

At the boundary $z=0$, these expressions approach the limits

$$
\begin{aligned}
\partial^{2} \chi / \partial z^{2} & =\int_{r}^{a} \frac{g_{1}(t) d t}{\left(t^{2}-r^{2}\right)^{1 / 2}} ; \quad 0 \leqslant r \leqslant a \\
& =0 ; \quad r>a \\
\partial^{3} \chi / \partial z^{3} & =-\frac{1}{r} \frac{d}{d r} \int_{0}^{\min (r, a)} \frac{t g_{1}(t) d t}{\left(r^{2}-t^{2}\right)^{1 / 2}},
\end{aligned}
$$

subject to suitable conventions concerning the signs of the square roots. These conventions are more fully discussed by Green [6].

It is convenient to represent the second harmonic function, $\psi$, as the sum of two functions, $\psi_{1}, \psi_{2}$, the first of which satisfies the boundary conditions

$$
\begin{aligned}
& \partial \psi_{1} / \partial z=u / 2(1-v) ; \quad 0 \leqslant r \leqslant b, \\
& \partial^{2} \psi_{1} / \partial z^{2}=0 ; \quad r>b .
\end{aligned}
$$

This function can be represented in the same form as $\chi$ above as

$$
\partial^{2} \psi_{1} / \partial z^{2}=\operatorname{Im} \int_{0}^{b} \frac{j(t) d t}{\left(r^{2}+(z-i t)^{2}\right)^{1 / 2}},
$$

On the boundary $z=0$, this simplifies to

$$
\begin{aligned}
\partial^{2} \psi_{1} / \partial z^{2} & =\int_{r}^{b} \frac{j(t) d t}{\left(t^{2}-r^{2}\right)^{1 / 2}} ; \quad 0 \leqslant r \leqslant b, \\
& =0 ; \quad r>b,
\end{aligned}
$$

thus satisfying 25 (ii) identically. 
The other boundary condition 25 (i) requires that

$$
\begin{aligned}
\left(\frac{d^{2} u}{d r^{2}}+\frac{1}{r} \frac{d u}{d r}\right) / 2(1-\nu) & =\left(\frac{\partial^{2}}{\partial r^{2}}+\frac{1}{r} \frac{\partial}{\partial r}\right) \frac{\partial \psi_{1}}{\partial z}=-\frac{\partial^{3} \psi_{1}}{\partial z^{3}}\left[\text { since } \nabla^{2} \partial \psi_{1} / \partial z=0\right] \\
& =\frac{1}{r} \frac{d}{d r} \int_{0}^{r} \frac{t j(t) d t}{\left(r^{2}-t^{2}\right)^{1 / 2}} ; \quad 0 \leqslant r \leqslant b
\end{aligned}
$$

by comparison with equation (24).

The surface displacement, $u$, is a prescribed function and hence equation (28) can be treated as an Abel integral equation for $j(r)$ whose solution is

$$
j(r)=\frac{1}{\pi(1-\nu) r} \frac{d}{d r} \int_{0}^{r} \frac{\left(t^{2} d u / d t\right) d t}{\left(r^{2}-t^{2}\right)^{1 / 2}} ; \quad 0 \leqslant r \leqslant b
$$

(see Copson [9] Lemma 2).

In that part of the surface where $r>b$,

$$
\frac{\partial^{3} \psi_{1}}{\partial z^{3}}=-\frac{1}{r} \frac{d}{d r} \int_{0}^{b} \frac{t j(t) d t}{\left(r^{2}-t^{2}\right)^{1 / 2}}
$$

Treating $\psi_{1}$, as a known function, the following boundary conditions remain to be satisfied by $\psi_{2}$ and $\chi$ :

$$
\begin{aligned}
& \partial \psi_{2} / \partial z=0 ; \quad 0 \leqslant r \leqslant b, \\
& \partial^{2} \chi / \partial z^{2}-\partial^{2} \psi_{2} / \partial z^{2}=-\frac{\alpha(1+\nu) T}{2(1-\nu)}+\frac{\partial^{2} \psi_{1}}{\partial z^{2}} ; \quad 0 \leqslant r \leqslant a, \\
& \partial^{3} \chi / \partial z^{3}-\partial^{3} \psi_{2} / \partial z^{3}=\partial^{3} \psi_{1} / \partial z^{3} ; \quad r \geqslant b .
\end{aligned}
$$

Condition 31(i) can be satisfied by representing $\partial \psi_{2} / \partial z$ in the form

$$
\frac{\partial \psi_{2}}{\partial z}=\operatorname{Im} \int_{b}^{\infty} g_{2}(t)\left\{\ln \left[\left(r^{2}+(z-i t)^{2}\right)^{1 / 2}+(z-i t)\right]+\frac{i \pi}{2}\right\} d t
$$

and the remaining two conditions will then give two simultaneous integral equations for the unknown functions $g_{1}, g_{2}$.

Equation (32) can be differentiated to give

$$
\begin{aligned}
& \frac{\partial^{2} \psi_{2}}{\partial z^{2}}=\operatorname{Im} \int_{b}^{\infty} \frac{g_{2}(t) d t}{\left(r^{2}+(z-i t)^{2}\right)^{1 / 2}}, \\
& \frac{\partial^{3} \psi_{2}}{\partial z^{3}}=\operatorname{Im}\left\{\frac{1}{r} \frac{d}{d r} \int_{b}^{\infty} \frac{(z-i t) g_{2}(t) d t}{\left(r^{2}+(z-i t)^{2}\right)^{1 / 2}}\right\}
\end{aligned}
$$

and, on the boundary $z=0$, these expressions reduce to

$$
\begin{aligned}
& \frac{\partial^{2} \psi_{2}}{\partial z^{2}}=\int_{\max (r, b)}^{\infty} \frac{g_{2}(t) d t}{\left(t^{2}-r^{2}\right)^{1 / 2}}, \\
& \begin{aligned}
\frac{\partial^{3} \psi_{2}}{\partial z^{3}} & =-\frac{1}{r} \frac{d}{d r} \int_{b}^{r} \frac{t g_{2}(t) d t}{\left(r^{2}-t^{2}\right)^{1 / 2}} ; \quad r>b \\
& =0 ; \quad 0 \leqslant r \leqslant b .
\end{aligned}
\end{aligned}
$$


We now substitute for $\partial^{2} \chi / \partial z^{2}, \partial^{2} \psi_{1} / \partial z^{2}, \partial^{2} \psi_{2} / \partial z^{2}$, from equations $(23,27,35)$ respectively into the boundary condition 28 (ii), obtaining

$$
\int_{b}^{\infty} \frac{g_{2}(t) d t}{\left(t^{2}-r^{2}\right)^{1 / 2}}+\int_{r}^{b} \frac{j(t) d t}{\left(t^{2}-r^{2}\right)^{1 / 2}}-\int_{r}^{a} \frac{g_{1}(t) d t}{\left(t^{2}-r^{2}\right)^{1 / 2}}=\frac{\alpha(1+\nu) T}{2(1-\nu)} ; \quad 0 \leqslant r \leqslant a .
$$

This can be treated as an Abel integral equation for $g_{1}$ whose solution is

$$
\begin{aligned}
g_{1}(x)=-\frac{2}{\pi} \frac{d}{d x} \int_{x}^{a}\left\{\int_{b}^{\infty} \frac{g_{2}(s) d s}{\left(s^{2}-t^{2}\right)^{1 / 2}}+\int_{t}^{b} \frac{j(s) d s}{\left(s^{2}-t^{2}\right)^{1 / 2}}-\frac{\alpha(1+\nu) T(t)}{2(1-\nu)}\right\} & \\
\times \frac{t d t}{\sqrt{t^{2}-x^{2}}} ; & 0 \leqslant x \leqslant a,
\end{aligned}
$$

see Copson [9], Lemma 3)

The order of integration in the last two terms can be changed to give

$$
\begin{aligned}
g_{1}(x)= & \frac{\alpha(1+\nu)}{\pi(1-\nu)} \frac{d}{d x} \int_{x}^{a} \frac{t T(t) d t}{\left(t^{2}-x^{2}\right)^{1 / 2}} \\
& -\frac{2}{x} \frac{d}{d x}\left\{\int_{x}^{b} j(s) \int_{x}^{\min (s, a)} \frac{t d t}{\left(t^{2}-x^{2}\right)^{1 / 2}\left(s^{2}-t^{2}\right)^{1 / 2}}\right. \\
& \left.+\int_{b}^{\infty} g_{2}(s) d s \int_{x}^{a} \frac{t d t}{\left(t^{2}-x^{2}\right)^{1 / 2}\left(s^{2}-t^{2}\right)^{1 / 2}}\right\} ; \quad 0 \leqslant x \leqslant a
\end{aligned}
$$

and on performing the inner integration this becomes

$$
\begin{aligned}
g_{1}(x)= & \frac{\alpha(1+\nu)}{\pi(1-\nu)} \frac{d}{d x} \int_{x}^{a} \frac{t T(t) d t}{\left(t^{2}-x^{2}\right)^{1 / 2}}+j(x) \\
& +\frac{2 x}{\pi\left(a^{2}-x^{2}\right)^{1 / 2}}\left\{\int_{a}^{b} \frac{\left(s^{2}-a^{2}\right)^{1 / 2} j(s) d s}{\left(s^{2}-x^{2}\right)}+\int_{b}^{\infty} \frac{\left(s^{2}-a^{2}\right)^{1 / 2} g_{2}(s) d s}{\left(s^{2}-x^{2}\right)}\right\} ; \quad 0 \leqslant x \leqslant a .
\end{aligned}
$$

The second integral equation, obtained from condition 31(iii) by substituting from equations $(24,30,36)$ is

$$
\frac{1}{r} \frac{d}{d r}\left\{\int_{b}^{r} \frac{t_{2}(t) d t}{\left(r^{2}-t^{2}\right)^{1 / 2}}+\int_{0}^{b} \frac{t j(t) d t}{\left(r^{2}-t^{2}\right)^{1 / 2}}-\int_{0}^{a} \frac{t g_{1}(t) d t}{\left(r^{2}-t^{2}\right)^{1 / 2}}\right\}=0 ; \quad r>b
$$

and it can be solved to give

$$
g_{2}(s)=\frac{2}{\pi s} \frac{d}{d s}\left\{\int_{b}^{s}\left\{B-\int_{0}^{b} \frac{t j(t) d t}{\left(r^{2}-t^{2}\right)^{1 / 2}}+\int_{0}^{a} \frac{t g_{1}(t) d t}{\left(r^{2}-t^{2}\right)^{1 / 2}}\right\} \frac{r d r}{\left(s^{2}-r^{2}\right)^{1 / 2}}\right\} ; \quad s \geqslant b
$$

where $B$ is a constant of integration to be determined from considerations of continuity at $s=b$.

On changing the order of integration and performing the inner integral, this 
becomes

$$
\begin{aligned}
g_{2}(s)= & \frac{2}{\pi\left(s^{2}-b^{2}\right)^{1 / 2}}\left\{B-\int_{0}^{b} \frac{t\left(b^{2}-t^{2}\right)^{1 / 2} j(t) d t}{\left(s^{2}-t^{2}\right)}\right. \\
& \left.+\int_{0}^{a} \frac{t\left(b^{2}-t^{2}\right)^{1 / 2} g_{1}(t) d t}{\left(s^{2}-t^{2}\right)}\right\}, \quad s \geqslant b .
\end{aligned}
$$

The constant $B$ is determined from the condition that temperature and hence $\partial^{2} \psi_{2} / \partial z^{2}$ should be continuous at $r=b$. This is only satisfied if $g_{2}(r)$ is bounded at $r=b$; hence

$$
B=\int_{0}^{b} \frac{t j(t) d t}{\left(b^{2}-t^{2}\right)^{1 / 2}}-\int_{0}^{a} \frac{\operatorname{tg}_{1}(t) d t}{\left(b^{2}-t^{2}\right)^{1 / 2}}
$$

and

$$
g_{2}(s)=\frac{2\left(s^{2}-b^{2}\right)^{1 / 2}}{\pi}\left\{\int_{0}^{b} \frac{t j(t) d t}{\left(s^{2}-t^{2}\right)\left(b^{2}-t^{2}\right)^{1 / 2}}-\int_{0}^{a} \frac{t g_{1}(t) d t}{\left(s^{2}-t^{2}\right)\left(b^{2}-t^{2}\right)^{1 / 2}}\right\} ; \quad s \geqslant b .
$$

This expression for $g_{2}(s)$ can now be substituted into equation (40) to give

$$
\begin{aligned}
g_{1}(x)= & \frac{4 x}{\pi^{2}\left(a^{2}-x^{2}\right)^{1 / 2}} \int_{b}^{\infty}\left\{\int_{0}^{b} \frac{t j(t) d t}{\left(s^{2}-t^{2}\right)\left(b^{2}-t^{2}\right)^{1 / 2}}\right. \\
& \left.-\int_{0}^{a} \frac{t g_{1}(t) d t}{\left(s^{2}-t^{2}\right)\left(b^{2}-t^{2}\right)^{1 / 2}}\right\} \frac{\left(s^{2}-a^{2}\right)^{1 / 2}\left(s^{2}-b^{2}\right)^{1 / 2} d s}{\left(s^{2}-x^{2}\right)} \\
& +\frac{\alpha(1+\nu)}{\pi(1-\nu)} \frac{d}{d x} \int_{x}^{a} \frac{t T(t) d t}{\left(t^{2}-x^{2}\right)^{1 / 2}}+j(x) \\
& +\frac{2 x}{\pi\left(a^{2}-x^{2}\right)^{1 / 2}} \int_{a}^{b} \frac{\left(s^{2}-a^{2}\right)^{1 / 2} j(s) d s}{\left(s^{2}-x^{2}\right)} ; \quad 0 \leqslant x \leqslant a,
\end{aligned}
$$

which can be expressed as a Fredholm integral equation of the second kind for $g_{1}(x)$ of the form

$$
g_{1}(x)=\int_{0}^{a} K(x, t) g_{1}(t) d t+f(x) ; \quad 0 \leqslant x \leqslant a,
$$

where

$$
\begin{aligned}
K(x, t)= & -\frac{4 x t}{\pi^{2}\left(b^{2}-t^{2}\right)^{1 / 2}\left(a^{2}-x^{2}\right)^{1 / 2}} \int_{0}^{\infty} \frac{\left(s^{2}-a^{2}\right)^{1 / 2}\left(s^{2}-b^{2}\right)^{1 / 2} d s}{\left(s^{2}-x^{2}\right)\left(s^{2}-t^{2}\right)}, \\
f(x)= & j(x)+\frac{2 x}{\pi\left(a^{2}-x^{2}\right)^{1 / 2}}\left\{\int_{a}^{b} \frac{\left(s^{2}-a^{2}\right)^{1 / 2} j(s) d s}{\left(s^{2}-x^{2}\right)}\right. \\
& \left.+\frac{2}{\pi} \int_{b}^{\infty} \int_{0}^{b} \frac{\left(s^{2}-a^{2}\right)^{1 / 2}\left(s^{2}-b^{2}\right)^{1 / 2} t j(t) d t d s}{\left(s^{2}-x^{2}\right)\left(s^{2}-t^{2}\right)\left(b^{2}-t^{2}\right)^{1 / 2}}\right\} \\
& +\frac{\alpha(1+\nu)}{\pi(1-v)} \frac{d}{d x} \int_{x}^{a} \frac{t T(t) d t}{\left(t^{2}-x^{2}\right)^{1 / 2}} .
\end{aligned}
$$

An iterative solution to equation (47) can be obtained if the ratio $a / b$ is small. 
In the physical problem, the radii of the perfect and imperfect contact areas, $a, b$, are not known a priori. One equation for determining these radii is obtained from the requirement that temperature be continuous at $r=a$. This condition is only satisfied if the terms involving $\left(a^{2}-x^{2}\right)^{-1 / 2}$ on the right hand side of equation (46) are self cancelling.

The second equation follows from the fact that the total compressive force on the punch, $P$, is a prescribed quantity. Using equations $(18,23)$, we have

$$
\begin{aligned}
P & =-\int_{0}^{a} 2 \pi r \sigma_{z z}(r, 0) d r=-4 \pi G \int_{0}^{a} \frac{\partial^{2} \chi}{\partial z^{2}}(r, 0) r d r \\
& =-4 \pi G \int_{0}^{a} \int_{r}^{a} \frac{r g_{1}(t) d t d r}{\left(t^{2}-r^{2}\right)^{1 / 2}} .
\end{aligned}
$$

On reversing the order of integration and performing the inner integral, this reduces to

$$
P=4 \pi G \int_{0}^{a} t g_{1}(t) d t
$$

It is also of interest to find the total heat flux through the contact area which is

$$
\begin{aligned}
Q & =\int_{0}^{b} 2 \pi r \frac{K \partial T}{\partial z}(r, 0) d r \\
& =\frac{4 \pi K(1-\nu)}{\alpha(1+\nu)} \int_{0}^{b}\left\{\frac{1}{2(1-\nu) r} \frac{d}{d r}\left(r \frac{d u}{d r}\right)-\frac{1}{r} \frac{d}{d r} \int_{0}^{\min (r, a)} \frac{\operatorname{tg}_{1}(t) d t}{\left(r^{2}-t^{2}\right)^{1 / 2}}\right\} r d r \\
& =\frac{2 \pi K}{\alpha(1+\nu)}\left\{b \frac{d u}{d r}(b)-2(1-\nu) \int_{0}^{a} \frac{\operatorname{tg}_{1}(t) d t}{\left(b^{2}-t^{2}\right)^{1 / 2}}\right\},
\end{aligned}
$$

from equations $(16,24,25,28)$.

\section{The spherical punch at uniform temperature}

We now consider in detail the case where the punch is a sphere of radius $R(\gg b)$ maintained at a uniform temperature $T_{0}$. The profile of such a punch is described by

$$
\frac{d u}{d r}=-\frac{r}{R}
$$

and hence

$$
j(r)=-\frac{1}{r} \frac{d}{d r} \int_{0}^{r} \frac{t^{3} d t}{\pi(1-\nu) R\left(r^{2}-t^{2}\right)^{1 / 2}}=-\frac{2 r}{\pi(1-\nu) R},
$$

from equation (29).

Substituting this result and $T=T_{0}$ (constant) into equation (49) and simplifying, 
we find

$$
\begin{aligned}
f(x)= & -\frac{\alpha(1+\nu) T_{0} x}{\pi(1-\nu)\left(a^{2}-x^{2}\right)^{1 / 2}}-\frac{2 x\left(b^{2}-x^{2}\right)^{1 / 2}}{\pi(1-\nu) R\left(a^{2}-x^{2}\right)^{1 / 2}} \\
& -\frac{4 x}{\pi^{2}(1-\nu) R\left(a^{2}-x^{2}\right)^{1 / 2}} \int_{b}^{\infty} \frac{\left(s-\left(s^{2}-a^{2}\right)^{1 / 2}\right)\left(s^{2}-b^{2}\right)^{1 / 2} d s}{\left(s^{2}-x^{2}\right)} .
\end{aligned}
$$

An iterative solution to equation (47) can now be found by expanding this expression in terms of the ratio of contact radii $(a / b)$. At each stage in the iteration process, the temperature $T_{0}$ is determined from the condition that the singular term in $\left(a^{2}-x^{2}\right)^{-1 / 2}$ be self-cancelling (see above, Section 7). Thus, for the purpose of the solution, $a / b$ is treated as an independent variable which determines the value of $T_{0}$.

The solution obtained is

$$
\begin{aligned}
& g_{1}(x)=\frac{-x\left(a^{2}-x^{2}\right)^{1 / 2}}{\pi(1-\nu) R b}\left\{1+\frac{x^{2}}{4 b^{2}}+\frac{a^{2}}{8 b^{2}}+\frac{x^{4}}{8 b^{4}}+\frac{a^{2} x^{2}}{8 b^{4}}+\frac{a^{4}}{16 b^{4}}+0\left(\frac{a^{6}}{b^{6}}\right)\right\}, \\
& T_{0}=\frac{-2 b}{\alpha(1+\nu) R}\left\{1-\frac{a^{2}}{4 b^{2}}-\frac{5 a^{4}}{64 b^{4}}-\frac{11 a^{6}}{256 b^{6}}+0\left(\frac{a^{8}}{b^{8}}\right)\right\} .
\end{aligned}
$$

Substituting these results into equation $(51,52)$ we find the total load

$$
P=\frac{\pi G b^{3}}{4(1-\nu) R}\left\{\frac{a^{4}}{b^{4}}+\frac{a^{6}}{4 b^{6}}+\frac{21 a^{8}}{128 b^{8}}+0\left(\frac{a^{10}}{b^{10}}\right)\right\}
$$

and the thermal contact conductance

$$
\frac{Q}{T_{0}}=\pi K b\left\{1+\frac{a^{2}}{4 b^{2}}+\frac{a^{4}}{64 b^{4}}+\frac{a^{6}}{256 b_{6}}+0\left(\frac{a^{8}}{b^{8}}\right)\right\} .
$$

A numerical solution has been developed which extends this procedure to terms of the order $(a / b)^{20}$. The results suggest that the first two terms in equations $(57-59)$ give an accuracy of $1 \%$ in the range $0 \leqslant a / b \leqslant 0.5$.

If the temperature $T_{0}$ is large and negative and the load $P$ is small, the ratio $a / b$ will become small and, in the limit, the outer radius $b$ of the imperfect contact region approaches

$$
b=-\alpha(1+\nu) T_{0} R / 2
$$

from the first term in equation (57).

The radius $a$, dividing the perfect from the imperfect contact region, can then be found by substituting into equation (58) and is approximately given by

$$
a=\left(\frac{-2 P \alpha T_{0} R^{2}\left(1-\nu^{2}\right)}{\pi G}\right)^{1 / 4} \text {. }
$$

The limiting value of the thermal contact conductance is

$$
\frac{Q}{T_{0}}=-\pi \alpha(1+\nu) T_{0} K R / 2
$$


It is of interest to compare these results with an extrapolation of the hot sphere result-i.e. a solution based on the assumption of a single circular region of perfect contact without regard to the consequent occurrence of tensile contact stresses when $T_{0}$ is negative (1). The radius obtained for this single region when $T_{0}$ is large and negative is

$$
b=-3 \alpha(1+\nu) T_{0} R / 2 \pi
$$

whilst the thermal contact conductance is

$$
\frac{Q}{T_{0}}=-6 \alpha(1+\nu) T_{0} K R / \pi .
$$

These results differ from equations $(60,62)$ in the ratios $1.05,1.22$ respectively.

\section{Conclusions}

The states of contact defined in equations (14) describe an idealization of a system with a pressure dependent thermal contact resistance which avoids the paradoxes incident to cooled indentation problens whilst retaining the desirable attribute of linearity in the boundary conditions of the resulting boundary value problems.

The example treated shows that such boundary value problems can be solved in simple cases and results are given for the indentation of a half-space by a cooled rigid sphere which extend the solution for the heated sphere previously obtained (1).

\section{Acknowledgements}

The author is very greatful to Professor J. Dundurs of Northwestern University for questioning the consistency of the notion of imperfect contact as here defined, thereby inducing the author to investigate it more deeply before committing himself to print.

\section{REFERENCES}

[1] Barber, J. R., Indentation of the semi-infinite elastic solid by a hot sphere, Int. J. Mech. Sci. 15 (1973) 813-819.

[2] Barber, J. R., The effect of heat flow on the contact area between a continuous rigid punch and a frictionless elastic half-space, Z. angew. Math. Phys. 27 (1976) 439-445.

[3] Dundurs, J. and Panek, C., Heat conduction between bodies with wavy surfaces, Int. J. Heat Mass Transfer 19 (1976) 731-736.

[4] Dundurs, J., Private communication.

[5] Williams, W. E., A solution of the steady state thermoelastic equations, $Z$. angew Math. Phys. 12 (1961) 452-455.

[6] Green, A. E., On Boussinesq's problem and penny-shaped cracks, Proc. Cambridge Phil. Soc. 45 (1949) 251-257.

[7] Green, A. E. and Zerna, W., Theoretical Elasticity, Clarendon Press, Oxford (1954) 174-178. 
[8] Collins, W. D., On the solution of some axisymmetric boundary value problems by means of integral equations, II Further problems for a circular disc and a spherical cap, Mathematika 6 (1959) 120-133.

[9] Copson, E. T., On the problem of the electrified disc, Proc. Edinburgh Math. Soc. 8 (1947) 14-19. 